\title{
The Effect of Different Movement Exercises on Cognitive and Motor Abilities
}

\author{
Monika Thomas \\ Institute of Physiology and Anatomy, German Sport University Cologne, Cologne, Germany \\ Email: thomas@dshs-koeln.de
}

Received June $15^{\text {th }}$, 2012; revised July $18^{\text {th }}$, 2012; accepted July $29^{\text {th }}, 2012$

\begin{abstract}
The influence of physical activity on motor and cognitive performance has been approved in several studies. However, it is still unclear which functions are affected, and why. It also remains unknown what type of physical training is best suitable. The present study focuses on special movement aspects based on the Brain Gym ${ }^{\circledR}$ program. Four groups of subjects $(n=64)$ participated in two experiments with pre-post intervention design. In experiment 1 , two groups of subjects were exposed to a sensorimotor adaptation study design by executing center out pointing movements under distorted visual feedback conditions with their dominant and non-dominant arm to test for intermanual transfer (IMT) as pre- and posttest. The intervention in both groups consisted of specified movement exercises with the right and left extremities: participants of Experimental group executed movements crossing the body midline and participants of Control group movements without crossing the body midline. Results showed a decreased retention of adaptation but larger IMT for Experimental group during posttest. We conclude that movements crossing the body midline impede retention but enhance IMT of sensorimotor adaptation. A potential relationship to an improvement of communication between the cerebral hemispheres evoked by the movement exercises crossing the body midline is rather speculative. In experiment 2, two groups were exposed to the $\mathrm{d} 2$-test measuring concentration and attention and a dice-test testing for visual-spatial abilities as pre- and posttest. The interventions were similar to experiment 1 . Results yielded no differences between groups such that different effects of both interventions could not have been shown.
\end{abstract}

Keywords: Motor Learning; Sensorimotor Adaptation; Cognition; Brain Gym ${ }^{\circledR}$

\section{Introduction}

The influence of physical activity on motor and cognitive performance has been investigated in many studies showing inconsistent results. Several studies could demonstrate a positive effect of physical activity on cognitive performance (Abeele \& Bock, 2001; Budde, Voelcker-Rehage, Pietrabyk-Kendziorra, Ribeiro, \& Tidow, 2008; Colcombe \& Kramer, 2003; Colcombe, Kramer, Erickson, Scalf, McAuley, Cohen, et al., 2004; Hillman, Pontifex, Raine, Castelli, Hall, \& Kramer, 2009; Planinsec, 2002; Voelcker-Rehage, Godde, \& Staudinger, 2011), while others fail to show facilitating effects (Blumenthal, Emery, Madden, Schniebolk, Walsh-Riddle, George, et al., 1991; Hill, Storandt, \& Malley, 1993). However, despite of this inconsistency, a meta-analysis of studies considering older subjects could show that indeed physical activity lead to an improvement of various cognitive abilities, especially executive functions (Colcombe \& Kramer, 2003). Beneath the influence on cognitive performance a few studies investigated the influence of physical activity on motor skill acquisition and found beneficial effects for different age groups (Hillman, Weiss, Hagberg, \& Hatfield, 2002; Mierau, Schneider, Abel, Askew, Werner, \& Struder, 2009; Richards, 1968). To date, most of the existing studies focused on the influence of cardiovascular fitness on cognitive or motor performance and learning (Colcombe, Kramer, Erickson, Scalf, McAuley, Cohen, et al., 2004; Hillman, Erickson, \& Kramer, 2008; Hillman, Pontifex, Raine, Castelli, Hall, \& Kramer, 2009; Hillman, Weiss, Hagberg, \&
Hatfield, 2002; Kramer \& Erickson, 2007; Voelcker-Rehage, Godde, \& Staudinger, 2011), only a few studies considered the influence of other activities like motor fitness or motor coordination (Budde, Voelcker-Rehage, Pietrabyk-Kendziorra, Ribeiro, \& Tidow, 2008; Planinsec, 2002; Voelcker-Rehage, Godde, \& Staudinger, 2011). Voelcker-Rehage and colleagues were able to show that cognitive functions in old age could be improved by both, cardiovascular and motor fitness (Voelcker-Rehage, Godde, \& Staudinger, 2010; Voelcker-Rehage, Godde, \& Staudinger, 2011). Additionally, the latter study revealed that both interventions lead to different changes in brain activity. Further studies which concentrated on motor coordination focused on children and young students yielding positive effects of coordinative abilities and coordination training on different cognitive abilities such as attention and reading comprehension skills (Budde, Voelcker-Rehage, Pietrabyk-Kend-ziorra, Ribeiro, \& Tidow, 2008; Planinsec, 2002; Uhrich \& Swalm, 2007). These effects became obvious even for a single training period of only 10 minutes (Budde, Voelcker-Rehage, Pietrabyk-Kendziorra, Ribeiro, \& Tidow, 2008). The influence of coordination training on motor performance has not been investigated so far.

Summing up, several results suggest that not only cardiovascular fitness but also coordinative training showed positive effects on cognitive functioning in older adults as well as in children and young students. Furthermore, several motor programs were developed which are said to support cognitive and motor performance. One program is called Brain Gym ${ }^{\circledR}$ devel- 
oped by Paul Dennison in 1981. This program consists of 26 simple movement exercises. They include movements of the extremities crossing the body midline in different ways. It is supposed that thereby an improvement of the connectivity between the right and left cerebral hemisphere can be obtained. In fact, due to missing evidences this idea is hardly challenged. Indeed, bimanual movement coordination is associated with interhemispheric communication (Andres, Mima, Schulman, Dichgans, Hallett, \& Gerloff, 1999; Serrien, 2009; Serrien \& Brown, 2002). However, it is disputable, if moving extremities across the body midline does evoke different effects than moving in the respective hemispace of the body.

The aim of this study was to investigate the influence of different movement patterns on motor abilities and cognitive functioning. Therefore, the influence of movement exercises crossing the body midline which are a part of the Brain Gym ${ }^{\circledR}$ program was compared to movement exercises not crossing the body midline. In a first experiment motor abilities were investigated by a sensorimotor adaptation and intermanual transfer task. In a second experiment cognitive abilities were measured using a concentration and attention test and a visuo-spatial skill test.

\section{Experiment 1 (Motor Performance)}

\section{Methods}

32 right-handed female subjects (19 to 30 years of age) participated in this experiment. None of them had overt sensorimotor dysfunctions except corrected vision, and none had prior experience with adaptation research. All signed an informed consent statement before participating in this study, which was part of an experimental program pre-approved by the authors' institutional Ethics Committee.

Subjects were subdivided into an experimental and a control group. All subjects participated in a sensorimotor adaptation test which was intermitted by a break of 7 - 10 days and an intervention phase (Table 1). While the sensorimotor adaptation test was the same for both subject groups the intervention phase differed between groups.

\section{Sensorimotor Adaptation Test}

As shown in Figure 1, subjects were seated in front of a table viewing a computer screen. On the right and left hand side of the subject a joystick was positioned such that subjects could handle it comfortably with elongated arms. Depending on the condition subjects either used the right or the left joystick, which position was represented as a cursor (C) on the computer screen. Additionally eight targets ( $\mathrm{T}$ ) (red dots, $1 \mathrm{~cm}$ diameter), positioned equidistantly on the circumference of a circle, were presented on the screen in a randomized order alternating with illumination of a starting position (St) (red dot, $1 \mathrm{~cm}$ diameter) in the center of the circle (Figure 1). Subjects' task was to execute center out pointing movements from the starting position to the different target positions. Subjects were instructed to move fast and accurately. The amplitude between the starting position and the targets was $10 \mathrm{~cm}$. A target was visible for 700 ms followed by presentation of the starting position which was illuminated until the cursor reached it triggering the illumination of the next target.

The sensorimotor adaptation test was separated into two test phases (pretest and posttest) which were interrupted by a break
Table 1.

Procedure of the sensorimotor adaptation test.

\begin{tabular}{|c|c|c|c|c|c|c|c|}
\hline \multirow[b]{3}{*}{ joystick } & \multicolumn{3}{|c|}{ Pretest } & \multirow[b]{3}{*}{$\begin{array}{c}\text { Break } \\
7-10 \text { days }\end{array}$} & \multirow[b]{3}{*}{$\begin{array}{l}\text { Intervention } \\
10 \mathrm{~min}\end{array}$} & \multicolumn{2}{|c|}{ Posttest } \\
\hline & base & line & $\mathrm{ADA}$ & & & $\mathrm{ADA}$ & IMT \\
\hline & right & left & $\overline{\text { right }}$ & & & right & left \\
\hline rotation & $0^{\circ}$ & $0^{\circ}$ & $60^{\circ}$ & & & $60^{\circ}$ & $60^{\circ}$ \\
\hline episodes & 3 & 3 & 3 & & & 15 & 5 \\
\hline
\end{tabular}

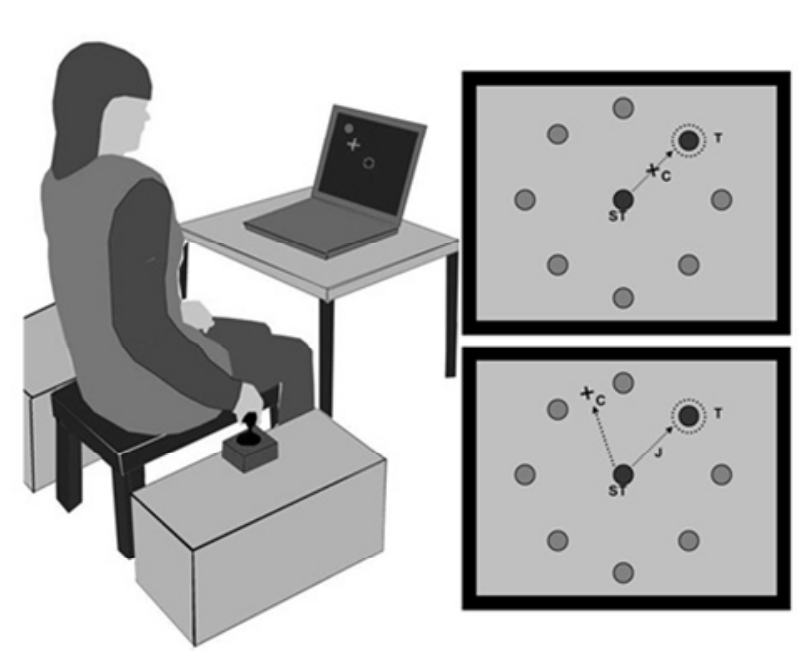

Figure 1.

Scheme of the experimental setup, with screen and joystick which can be placed on the right and left hand side of the subject. Top right: Subjects' view under baseline condition with computer-generated targets (T) in 8 possible directions (only one target visible at a given time) and starting position (ST). Cursor position (C) represented joystick position. Bottom right: Subjects view under adaptation condition and IMT where the joystick (J) and cursor position were shifted about $60 \mathrm{deg}$.

and the intervention phase (see below) executed immediately before the posttest. Pre- and post-test were subdivided into episodes including 8 pointing movements each, thus one movement to each target. The target sequence was randomized between episodes.

The pretest consisted of 9 episodes starting with a baseline measurement of 3 episodes using the right joystick followed by 3 episodes using the left joystick where the cursor position matched the joystick position. The last 3 episodes were executed with the right joystick and the cursor position was $60^{\circ}$ rotated in counter-clockwise direction with respect to the joystick position to induce adaptation. Again, subjects' task was to move the cursor to the target position implying that the joystick had to be moved in clockwise direction.

The posttest consisted of 20 episodes starting with 15 episodes under the same condition as the last 3 episodes of the pretest thus continuing the adaptation. During the last 5 episodes cursor position was again $60^{\circ}$ rotated in counter clockwise direction by using the left joystick to test for intermanual transfer of adaptation (Table 1).

\section{Intervention}

Subjects executed two different movement exercises for 10 minutes. Depending on group affinity, subjects moved their 
limbs across the body midline (experimental group) or moved their limbs in the ipsilateral hemispace (control group). On an instruction video a female exercise instructor was shown who executed the movement exercises slowly. A male voice simultaneously explained the exercises and instructed the subjects to imitate.

1) Exercises of the experimental group (movements across the body midline): The subjects practiced two different movements which were part of the "midline movements" of the Brain $\mathrm{Gym}^{\circledR}$ exercise program. At the beginning subjects were instructed to situate comfortable in an upright position. For the first exercise ("cross crawl") they were instructed to move the right elbow in direction of the left knee and simultaneously move the knee in the direction of the elbow such that the right elbow and the left knee touched each other at the outer edges. After completing this movement, subjects had to bring their limbs back into the starting position and repeat the movement with the other side. Subjects were requested to execute 3 series with 10 movements of each side, simultaneously with the video counting the number. During the first series the movements were shown on the video simultaneously. During the second series, subjects had to fixate an $\mathrm{X}$ which was shown on the video and during the third series subjects had to close their eyes and imagine the $\mathrm{X}$ which they have seen before while moving. For the second exercise ("lazy eights") subjects were instructed to stretch the right arm in front with the thumb up. Subjects were requested to trace a recumbent eight in front of their body with the center in the middle at eye level starting the trace right above. Subjects should track their thumb with the eyes without moving the head. Subjects executed 3 series tracing 6 recumbent eights. In the first series they moved with the right hand, in the second series with the left hand and the third series with both hands simultaneously. Movements were as well shown on the video counting the repetitions.

2) Exercises of the control group (movements in the ipsilateral hemispace): Subjects of the control group practiced two different movement exercises which were not part of the Brain $\mathrm{Gym}^{\circledR}$ exercise program but were used as "counterparts" to the "midline-movements". Like in the experimental group subjects were instructed to situate comfortable in an upright position. For the first exercise they were instructed to move the right elbow in direction of the right knee and simultaneously move the knee in the direction of the elbow such that the right elbow and the right knee touched each other. After completing the movement, subjects had to bring their limbs back into the starting position and proceed with the opposite side. Subjects were requested to execute 3 series with 10 movements on each side. Again, the video simultaneously counted the repetitions. During the first series the movements were shown on the video screen. During the second series subjects, had to fixate two parallel lines (II) while moving and during the third series subjects had to close their eyes and imagine the parallel lines. For the second exercise subjects were instructed to stretch the right arm in front with the thumb up. Subjects were requested to trace a circle in front of their body which was not allowed to cross the body midline starting the trace right above. The thumb should have been tracked with the eyes without moving the head. Subjects executed 2 series tracing 9 circles. During the first series they moved with their right hand in the right hemispace and during the second series they moved with their left hand in the left hemispace. Again, movements were executed on the video counting the number.

\section{Statistical Analyses}

To quantify the performance of sensorimotor adaptation we calculated the initial error as the angle between joystick and target direction $150 \mathrm{~ms}$ after movement onset as a parameter which is unconfounded by feedback-based corrections. Additionally, we determined end point accuracy as the distance between target position and end position of the joystick. For further analyses, we calculated the mean values of initial error and endpoint accuracy for each episode. Each variable was submitted to analyses of variances (ANOVAs) separately for adaptation phase with the right hand (ADA) and adaptation phase with the left hand (IMT). ADA was analyzed by ANOVAs with the within factors episode and block and the between factor group. One block consists of 3 episodes. IMT was analyzed by ANOVAs with the within-factor episode and the between factor group. We additionally calculated the difference between the last adaptation episode of pretest and the first adaptation episode of posttest for both parameters to evaluate the influence of intervention on consolidation of adaptation and submitted the outcome to t-tests.

\section{Results}

Figure 2 illustrates the initial error $150 \mathrm{~ms}$ after movement onset averaged across subjects separately for each episode and group for the adaptation phase (ADA) and the intermanual transfer phase (IMT). At the beginning of adaptation phase, subjects of both groups started with an error of about $35^{\circ}$. During the first three episodes the error decreased gradually in both groups. In the first episode of the posttest the experimental group started with a distinct greater initial error than in the last episode of the pretest while the control group reached a comparable level in both episodes. During the following adaptation phase the initial error decreased in both groups reaching a similar level of about $6^{\circ}$ at the end. Accordingly, ANOVA yielded significant effects of episode and block and the interaction of both. Group effects were not significant (Table 2). The inset of Figure 2 depicts the difference between the last adaptation episode of pretest and the first adaptation episode of post

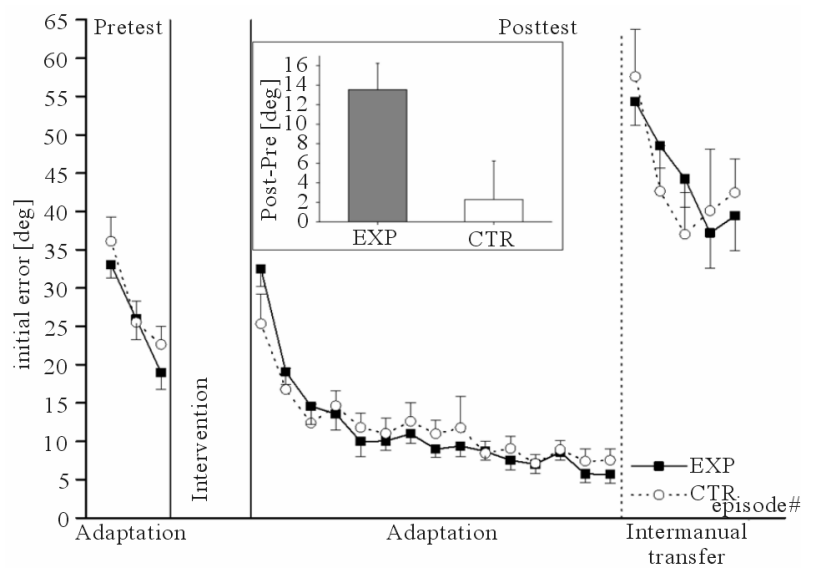

Figure 2.

Time course of initial error during the adaptation phase (ADA) of preand posttest and the intermanual transfer phase (IMT) separated for each group. Symbols represent across-subject means, and error bars standard errors. Inset: Across-subject means of the difference between the last adaptation episode of pretest and the first adaptation episode of posttest. 


\section{THOMAS}

Table 2.

Results of analyses of variances of the adapation phase (ADA) and the intermanual transfer phase (IMT) for the parameters initial error and endpoint accuracy.

\begin{tabular}{|c|c|c|c|c|}
\hline & \multicolumn{2}{|c|}{ Initial error } & \multicolumn{2}{|c|}{ Endpoint accuracy } \\
\hline & $\mathrm{ADA}$ & IMT & $\mathrm{ADA}$ & IMT \\
\hline Episode & $\mathrm{F}_{(2,58)}=59.17^{* * *}$ & $F_{(4,116)}=12.08^{* * *}$ & $F_{(2,58)}=68.83^{* * * *}$ & $F_{(4,116)}=9.78^{* * *}$ \\
\hline Block & $\mathrm{F}_{(5,145)}=53.38^{* * *}$ & - & $\mathrm{F}_{(5,145)}=43.07^{* * *}$ & - \\
\hline Episode block & $\mathrm{F}_{(10,290)}=15.14^{* * *}$ & - & $\mathrm{F}_{(10,290)}=15.55^{* * * *}$ & - \\
\hline Group & $\mathrm{F}_{(1,29)}=0.1$ & $\mathrm{~F}_{(1,29)}=0.01$ & $\mathrm{~F}_{(1,29)}=1.53$ & $F_{(1,29)}=4.3^{*}$ \\
\hline Group* episode & $\mathrm{F}_{(2,58)}=0.51$ & $\mathrm{~F}_{(4,116)}=1.73$ & $\mathrm{~F}_{(2,58)}=0.45$ & $\mathrm{~F}_{(2,58)}=1.14$ \\
\hline Group*block & $\mathrm{F}_{(5,145)}=1.1$ & - & $\mathrm{F}_{(5,145)}=0.42$ & - \\
\hline Group*episode*block & $F_{(10,290)}=0.81$ & - & $\mathrm{F}_{(10,290)}=0.8$ & - \\
\hline
\end{tabular}

test showing a greater difference for the experimental group than the control group which was statistically significant $(\mathrm{t}(29)=$ $2.28 ; p<0.05)$.

At the onset of the intermanual transfer phase the error increased for both groups. During the following episodes, the error decreased similar for both groups. ANOVA yielded a significant effect of episode and no significant group effects (Table 2).

Figure 3 depicts the endpoint accuracy in the same manner as for the initial error. Adaptation course is comparable to one shown with the initial error. Accordingly, ANOVA again yielded significant effects of episode and block and the interaction of both. Group effects were not significant (Table 2). The inset of Figure 2 shows that the difference between the end of adaptation of the pretest and the onset of adaptation of the posttest was less different between groups than for the initial error not reaching statistical significance $(\mathrm{t}(29)=0.72 ; p>$ 0.05).

Endpoint accuracy decreased distinctly for both groups at the

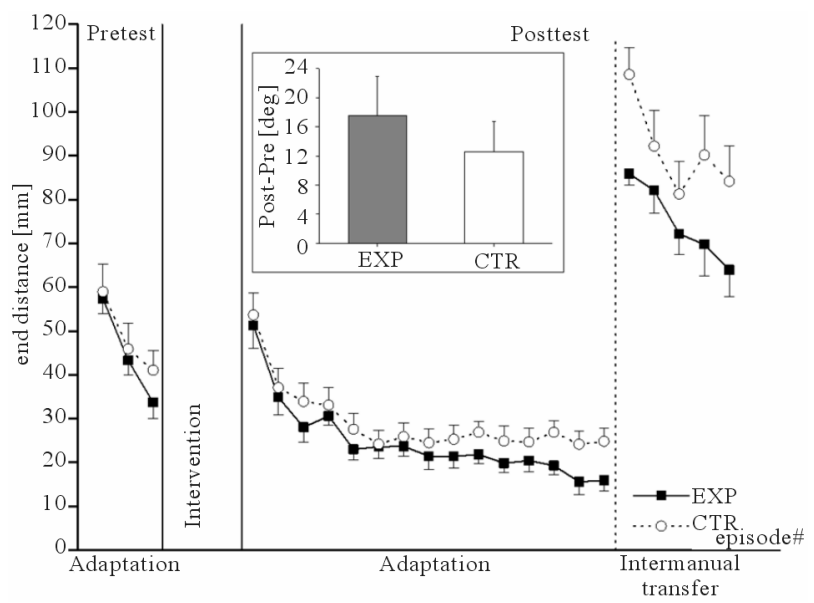

Figure 3.

Time course of end distance during the adaptation phase (ADA) of preand posttest and the intermanual transfer phase (IMT) separated for each group. Symbols represent across-subject means, and error bars standard errors. Inset: Across-subject means of the difference between the last adaptation episode of pretest and the first adaptation episode of posttest. onset of the intermanual transfer phase. This decrease was more pronounced in the experimental group. The difference between both groups persists during all 5 episodes of the intermanual transfer phase. Accordingly, ANOVA showed a significant effect of episode and group (Table 2).

\section{Discussion}

In the present study we investigated whether special movement patterns with the right and left extremities have an impact on sensorimotor adaptation and the intermanual transfer of adaptation from the dominant right to the left hand. Therefore, subjects executed specified movement exercises with the right and left extremities as intervention. Depending on group affinity, subjects executed movements crossing the body midline (experimental group) or moved only in the respective hemispace without passing the midline of the body (control group). We found no differences during the time course of adaptation between groups for both parameters, the initial error and the end point accuracy. Thus, different effects on the learning rate of the different movement performances could not be shown. However, for the initial error the difference between the last episode of pretest and the first episode of posttest was significant lower for the control group, while there was no group difference for the end point accuracy. It is known that adaptive improvement persists in the sensorimotor system over time which is called consolidation (Abeele \& Bock, 2001; McGaugh, 2000; Shadmehr \& Holcomb, 1997). It has been shown that subjects could recall the learned behavior even after breaks of up to one month (Bock, Schneider, \& Bloomberg, 2001). Our results indicate consolidation of adaptation only for the control group, where the initial error at the onset of posttest was similar to the error at the end of the pretest. In contrast, for the experimental group, the initial error at the onset of posttest was similar to the error at the onset of pretest, thus revealing no consolidation of the learned behavior. In relation to the studies mentioned above, our results suggest that the movement performance crossing the body midline impede consolidation, while the ipsilateral movement pattern does not. In conjunction with the idea that bilateral movements crossing the body midline facilitate the connectivity between both brain hemispheres, our results imply that such reorganization between the hemispheres could have a negative effect on retention of the learned 
motor behavior.

For intermanual transfer the effects were different compared to the retention of learning: group effects have been shown for end point accuracy but were absent for the initial error. The significant group effect indicates that movement performance crossing the body midline has a beneficial effect on the intermanual transfer of the accuracy at the end of movements. Referring to the statement mentioned above, this observation suggests that an enhancement of the connectivity between the cerebral hemispheres can improve access to the adaptation information of the opposite arm.

Obviously, different parameters reveal significant group effects for retention of learning and the intermanual transfer. Previous studies indicated that the dominant arm shows a benefit in controlling the initial movement direction while the non-dominant arm is more proficient in controlling the final posture (Sainburg, 2002; Sainburg \& Kalakanis, 2000). Relating these results to the results of our study, it may be accepted that basically the dominant aspect of movement control of the respective arm can be affected by the intervention. In consequence, the control of initial movement trajectory should be the affected movement aspect. For intermanual transfer, further findings supported that opposite arm training improves the end point accuracy of the left arm, whereas opposite arm training improves the initial movement direction of the right arm (Sainburg \& Wang, 2002). Therefore, it is conceivable that an impact of the intervention has been found on the end point accuracy rather than on the initial movement direction.

To sum up, the present results refer to an impairment of retention of the adaptive behavior, when preceding movement exercises across the body midline. In contrast, the same movement exercises seem to enhance intermanual transfer. A causal relation of these results with an enhancement of the cerebral connectivity is merely speculative.

\section{Experiment 2 (Cognitive Performance)}

\section{Methods}

Again, 32 right-handed female subjects (19 to 30 years of age) participated in this experiment. All were naive regarding the cognitive tests used in this experiment. All signed an informed consent statement before participating in this study, which was part of an experimental program pre-approved by the authors' institutional Ethics Committee.

Subjects were subdivided into an experimental and a control group. All subjects participated in two cognitive tests as pretest followed by a break of 7 - 10 days and an intervention phase (Table 2) and again in the same cognitive tests as posttest. The interventions were exactly the same as in experiment 1 (see above).

\section{Cognitive Tests}

To test subjects' cognitive performance two neuropsychological tests were used in a pre-post design including the domains attention and concentration using the d2-attention-test (Brickenkamp, 2002) and visuo-constructive skills by the dice-subtest of the I-S-T 2000R (Liepmann et al., 2007). The d2-attention-test is a paper-pencil test implicating a letter cancellation task. It consists of 14 lines of 47 randomly mixed "d" and "p" each. The letters are arranged with different numbers of dashes (min. 0 dashes-max. 4 dashes). Subjects were in- structed to score out every "d" which was arranged with two dashes, either individually or in pairs. The time for each line was limited to $20 \mathrm{~s}$. The whole test lasted $4.67 \mathrm{~min}$. The test-retest reliability of the d2-test of attention has been indicated with 0.95 - 0.98. For the dice-test 5 different dices with 6 different patterns each were presented on a paper while three patterns were visible. Additionally, 10 dices where presented which show one of the given 5 dices in a modified position. Subjects' task was to find out the appropriate given dice for each of the 10 dices. This task was repeated once with 5 other given dices. Overall, the task lasted $9 \mathrm{~min}$. The test-retest reliability has not been evaluated.

\section{Statistical Analyses}

For the d2-attention-test the number of correct responses minus errors of confusion (KL) was calculated for each subject separately for the pre- and posttest. The KL-value is defined as an objective measure of concentration (Brickenkamp, 2002). For the dice-test the number of correct responses has been transformed into standard values (SV) according to norm groups. The outcomes of both were submitted to analyses of variance with the between factor group and the within-factor time (pre/post).

\section{Results}

Figure 4(a) shows the KL values of the d2-test averaged across subjects of the experimental group and the control group in the pre- and posttest. Both groups improved their performance, thus ANOVA yielded a significant effect of time $(\mathrm{F}(1,30)=$ 72.98; $p=0.000)$. Group effect and the interaction between group and time were not significant (group: $\mathrm{F}(1,30)=0.02 ; p=$ 0.888 ; group ${ }^{*}$ time: $\left.\mathrm{F}(1,30)=0.01 ; p=0.926\right)$.

Figure 4(b) shows the SV values of the dice-test averaged across subjects of experimental group and control group in the pre- and post-test. Again, both groups improved their performance yielding a significant effect of time $(\mathrm{F}(1,30)=15.52 ; p=$ $0.000)$. The factor group and the interaction of group and time were not significant (group: $\mathrm{F}(1,30)=0.18 ; p=0.671$; group ${ }^{*}$ time: $\left.\mathrm{F}(1,30)=1.96 ; p=0.171\right)$.

\section{Discussion}

The aim of the second experiment was to investigate whether movement exercises crossing the body midline have a different effect on attention, concentration and visual-spatial skills than moving extremities in the ipsilateral hemispace of the body. For both, the d2-test (concentration and attention) and the dice-test (visuo-spatial skills), group differences were not statistically significant. Hence, different influences of the respective movement patterns could not be confirmed. However, the posttest results reveal a significant increase of performance for both tests. This lets suggest, that both movement patterns were equally effective. For the d2-test strong learning effects are not likely because the test-retest reliability is extraordinarily high (see Methods). For the dice-test the test-retest reliability has not been verified such that a learning effect cannot be excluded so far. In conclusion, a benefit of movements across the body midline over movements in the ipsilateral hemispace for the abilities of concentration, attention and visuo-spatial skill cannot be confirmed. In fact, it seems that the movement patterns of both 


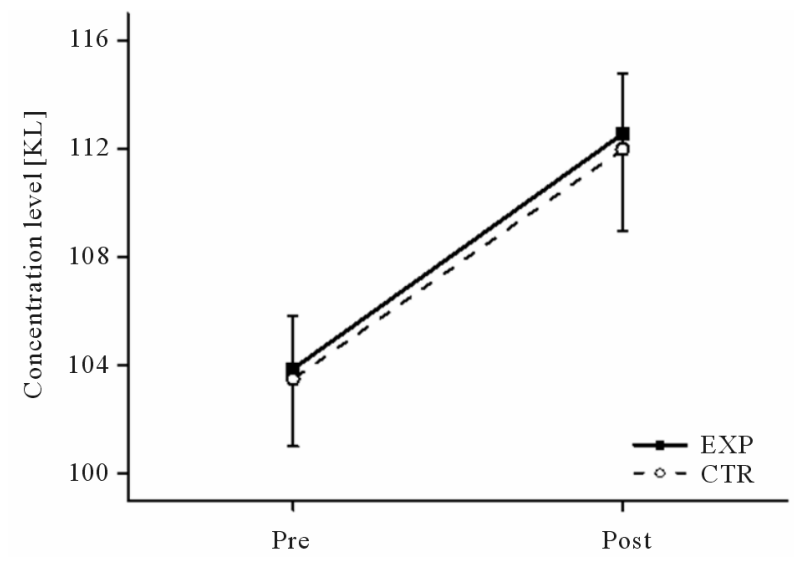

(a)

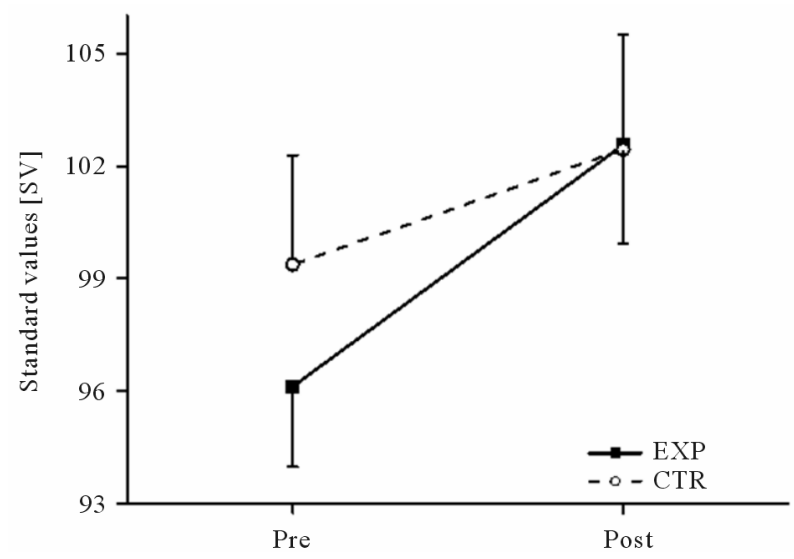

(b)

Figure 4.

(a) Concentration level $(\mathrm{KL})$ of the d2-attention test of the pre- and post-test for experimental group (EXP) and control group (CTR). Symbols represent across-subject means, and error bars standard errors; (b) Standard values of the dice-test separated for each group.

groups improve concentration and attention in an equal manner, while for the visuo-spatial abilities the situation is ambiguous.

\section{General Discussion}

The aim of the study was to present a first overview on new aspects concerning the influence of physical activity on motor and cognitive abilities, investigating special features of movement performance using exercises from the Brain Gym ${ }^{\circledR}$ program. As mentioned in the introduction, Brain $\mathrm{Gym}^{\circledR}$ is based on the idea that movements crossing the body midline enhance the communication of the cerebral hemispheres. Indeed, for the sensorimotor adaptation task movements across the body midline affect adaptation and intermanual transfer in a different way than movement performance in the ipsilateral hemispace of the body. This difference could not be confirmed for the tested cognitive abilities (concentration, attention and visuo-spatial abilities). Nevertheless, for several cognitive abilities, such as executive functions, interhemispheric communication plays an important role (Sauseng, Klimesch, Schabus, \& Doppelmayr, 2005; Shibata, Shimoyama, Ito, Abla, Iwasa, Koseki, et al., 1997; Shibata, Shimoyama, Ito, Abla, Iwasa, Koseki, et al., 1998). Beside numerous intrahemispheric connections, visuo- spatial abilities tested by mental rotation tasks, require interhemispheric associations as well (Bhattacharya, Petsche, Feldmann, \& Rescher, 2001); the neural processes associated with attention are rather ambiguous. It is debatable, whether long-time interventions could possibly result in different effects of both interventions even for cognitive abilities and lead to stronger effects at all. However, this study is limited in the fact that sound arguments for the differences in influencing motor performance and cognitive abilities cannot be concluded. Summing up, a positive effect of the Brain Gym $^{\circledR}$ exercises in contrast to the ipsilateral movement patterns cannot be confirmed entirely, although serious differences become obvious. This issue requires further empirical study.

\section{Acknowledgements}

Thanks are due to Dipl.-Ing. (FH) L. Geisen for software development and L. Bock, N. Feidt and S. Bethke for data collection and analysis. Responsibility for the contents rests with the author.

\section{REFERENCES}

Abeele, S., \& Bock, O. (2001). Mechanisms for sensorimotor adaptation to rotated visual input. Experimental Brain Research, 139, 248 253. doi: $10.1007 / \mathrm{s} 002210100768$

Andres, F. G., Mima, T., Schulman, A. E., Dichgans, J., Hallett, M., \& Gerloff, C. (1999). Functional coupling of human cortical sensorimotor areas during bimanual skill acquisition. Brain, 122, 855-870. doi:10.1093/brain/122.5.855

Bhattacharya, J., Petsche, H., Feldmann, U., \& Rescher, B. (2001). Eeg gamma-band phase synchronization between posterior and frontal cortex during mental rotation in humans. Neuroscience Letters, 311, 29-32. doi:10.1016/S0304-3940(01)02133-4

Blumenthal, J. A., Emery, C. F., Madden, D. J., Schniebolk, S., Walsh-Riddle, M., George, L. K., et al. (1991). Long-term effects of exercise on psychological functioning in older men and women. Journals of Gerontology, 46, 352-361.

Bock, O., Schneider, S., \& Bloomberg, J. (2001). Conditions for interference versus facilitation during sequential sensorimotor adaptation. Experimental Brain Research, 138, 359-365.

doi:10.1007/s002210100704

Brickenkamp, K. (2002). Test d2 aufmerksamkeits-belastungs-test, manual (The d2 test of attention). Göttingen-Bern-Toronto-Seattle: Hogrefe, Ltd.

Budde, H., Voelcker-Rehage, C., Pietrabyk-Kendziorra, S., Ribeiro, P. \& Tidow, G. (2008). Acute Coordinative Exercise Improves Attentional Performance in Adolescents. Neuroscience Letters, 441, 219223. doi:10.1016/j.neulet.2008.06.024

Colcombe, S., \& Kramer, A. F. (2003). Fitness effects on the cognitive function of older adults: A meta-analytic study. Psychological Science, 14, 125-130. doi:10.1111/1467-9280.t01-1-01430

Colcombe, S. J., Kramer, A. F., Erickson, K. I., Scalf, P., McAuley, E., Cohen, N. J., et al. (2004). Cardiovascular fitness, cortical plasticity, and aging. Proceedings of the National Academy of Sciences of the USA, 101, 3316-3321. doi:10.1073/pnas.0400266101

Hill, R. D., Storandt, M., \& Malley, M. (1993). The impact of longterm exercise training on psychological function in older adults. Journals of Gerontology, 48, 12-17.

Hillman, C. H., Erickson, K. I., \& Kramer, A. F. (2008). Be smart, exercise your heart: Exercise effects on brain and cognition. Nature Reviews Neuroscience, 9, 58-65. doi:10.1038/nrn2298

Hillman, C. H., Pontifex, M. B., Raine, L. B., Castelli, D. M., Hall, E. E., \& Kramer, A. F. (2009). The effect of acute treadmill walking on cognitive control and academic achievement in preadolescent children. Neuroscience, 159, 1044-1054.

doi:10.1016/j.neuroscience.2009.01.057 


\section{THOMAS}

Hillman, C. H., Weiss, E. P., Hagberg, J. M., \& Hatfield, B. D. (2002). The relationship of age and cardiovascular fitness to cognitive and motor processes. Psychophysiology, 39, 303-312. doi:10.1017/S0048577201393058

Kramer, A. F., \& Erickson, K. I. (2007). Capitalizing on cortical plasticity: Influence of Physical activity on cognition and brain function. Trends in Cognitive Sciences, 11, 342-348. doi:10.1016/j.tics.2007.06.009

Liepmann, D., Beauducel, A., Brocke, B., Amthauer, R. (2007). Intelligenz-struktur-test 2000R, manual (intelligence-structure-test). Göttingen-Bern-Toronto-Seattle: Hogrefe, Ltd.

McGaugh, J. L. (2000). Memory-A century of consolidation. Science, 287, 248-251. doi:10.1126/science.287.5451.248

Mierau, A., Schneider, S., Abel, T., Askew, C., Werner, S., \& Struder, H. K. (2009). Improved sensorimotor adaptation after exhaustive exercise is accompanied by altered brain activity. Physiology \& Behavior, 96, 115-121. doi:10.1016/j.physbeh.2008.09.002

Planinsec, J. (2002). Relations between the motor and cognitive dimensions of preschool girls and boys. Percept Mot Skills, 94, 415-423. doi:10.2466/pms.2002.94.2.415

Richards, D. K. (1968). A two-factor theory of the warm-up effect in jumping performance. Research Quarterly, 39, 668-673.

Sainburg, R. L. (2002). Evidence for a dynamic-dominance hypothesis of handedness. Experimental Brain Research, 142, 241-258. doi:10.1007/s00221-001-0913-8

Sainburg, R. L., \& Kalakanis, D. (2000). Differences in control of limb dynamics during dominant and nondominant arm reaching. Journal of Neurophysiology, 83, 2661-2675.

Sainburg, R. L., \& Wang, J. (2002). Interlimb transfer of visuomotor rotations: Independence of direction and final position information. Experimental Brain Research, 145, 437-447. doi:10.1007/s00221-002-1140-7

Sauseng, P., Klimesch, W., Schabus, M., \& Doppelmayr, M. (2005). Fronto-parietal eeg coherence in theta and upper alpha reflect Central executive functions of working memory. International Journal of Psychophysiology, 57, 97-103. doi:10.1016/j.ijpsycho.2005.03.018

Serrien, D. J. (2009). Bimanual information processing and the impact of conflict during mirror drawing. Behavioural Brain Research, 205, 391-395. doi:10.1016/j.bbr.2009.07.015

Serrien, D. J., \& Brown, P. (2002). The functional role of interhemispheric synchronization in the control of bimanual timing tasks. Experimental Brain Research, 147, 268-272. doi:10.1007/s00221-002-1253-Z

Shadmehr, R., \& Holcomb, H. H. (1997). Neural Correlates of Motor Memory Consolidation. Science, 277, 821-825. doi:10.1126/science.277.5327.821

Shibata, T., Shimoyama, I., Ito, T., Abla, D., Iwasa, H., Koseki, K., et al. (1997). The time course of interhemispheric eeg coherence during a go/no-go task in humans. Neuroscience Letters, 233, 117-120. doi:10.1016/S0304-3940(97)00652-6

Shibata, T., Shimoyama, I., Ito, T., Abla, D., Iwasa, H., Koseki, K., et al. (1998). The synchronization between brain areas under motor inhibition process in humans estimated by event-related eeg coherence. Neuroscience Research, 31, 265-271. doi:10.1016/S0168-0102(98)00046-7

Uhrich, T. A., \& Swalm, R. L. (2007). A pilot study of a possible effect from a motor task on reading performance. Perceptual \& Motor Skills Percept Mot Skills, 104, 1035-1041. doi:10.2466/pms.104.3.1035-1041

Voelcker-Rehage, C., Godde, B., \& Staudinger, U. M. (2010). Physical and motor fitness are both related to cognition in old age. European Journal of Neuroscience, 31, 167-176. doi:10.1111/j.1460-9568.2009.07014.x

Voelcker-Rehage, C., Godde, B., \& Staudinger, U. M. (2011). Cardiovascular and coordination training differentially improve cognitive performance and neural processing in older adults. Frontiers in $\mathrm{Hu}$ man Neuroscience, 5, 26. doi:10.3389/fnhum.2011.00026 